\title{
Learning with Data Streams - An NNTree Based Approach
}

\author{
Qiangfu Zhao \\ The University of Aizu, Aizuwakamatsu, Japan \\ qf-zhao@u-aizu.ac.jp \\ http://WWW.u-aizu.ac.jp/ qf-zhao
}

\begin{abstract}
The plasticity-stability dilemma is a key problem for learning with data streams. On the one hand, the learner must be plastic enough to adapt to the new data. On the other hand, it must be stable enough to integrate information. In this paper, we try to resolve this problem using neural network trees (NNTrees). An NNTree is a decision tree (DT) with each non-terminal node containing an expert neural network (ENN). The NNTrees are plastic because they can adapt to the new data through retraining of the ENNs and/or through generation of new nodes. The NNTrees are also stable because retraining is performed partially and locally. In this paper, we propose an algorithm that can grow NNTrees effectively and efficiently. Experiments with several public databases show that the NNTrees obtained by the proposed methods are comparable with the NNTrees or DTs obtained with all data provided all-at-once.
\end{abstract}

Keywords: Machine learning, neural networks, decision trees, on-line learning, neural network trees, plasticity-stability dilemma.

\section{Introduction}

The plasticity-stability dilemma is a key problem for learning with data streams. On the one hand, the learner must be plastic enough to adapt to the new data. On the other hand, it must be stable enough to integrate information. Usually, fully connected neural networks are plastic but not stable. To make the system stable, some kind of localization is necessary. In this paper, we try to resolve this problem using neural network trees (NNTrees). An NNTree is a decision tree (DT) with each non-terminal node containing an expert neural network (ENN). The basic structure of an NNTree is shown in Fig. 1, The NNTrees are plastic because they can adapt to the new data through retraining of the ENNs and/or through generation of new nodes. The NNTrees are also stable because retraining of the ENNs is performed partially and locally. Partial retraining means that the weights of the ENNs are updated only for a limited number of epochs. Local retraining means that, for each new datum, only the ENNs on the "search path" are modified.

Note that traditional DTs can also be induced using a data stream through restructuring of the tree [1]. The problem in this approach is that all information 


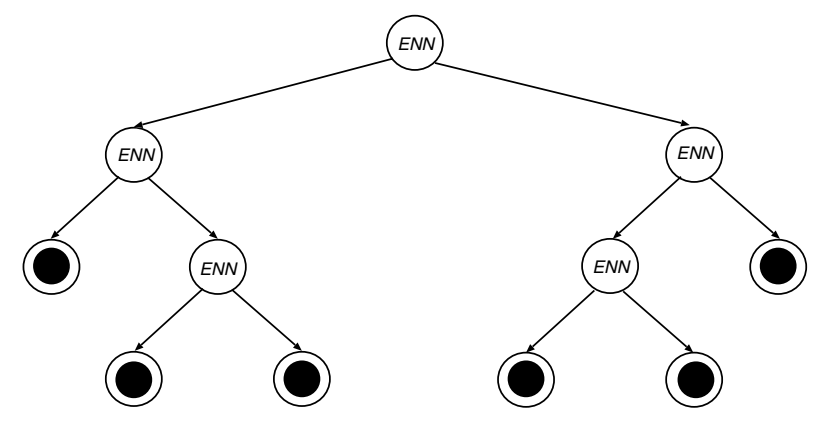

Fig. 1. Structure of an neural network tree

for restructuring the tree must be preserved. This is not possible if the duration of the data stream is very long. Using the NNTrees, however, we do not have to keep all information. As shown in this paper, a sliding window might be good enough to keep information for retraining the ENNs or for generating new nodes.

The main contribution of this paper is to propose an NNTree based algorithm for learning with data streams. The basic idea of this algorithm is to retrain the ENNs to adapt to new data. Retraining is performed partially in two senses. First, only a limited number of epochs are used in retraining. Second, only a smaller number of data kept in a sliding window are used in retraining. New nodes can be generated when retraining alone is not enough. This paper is organized as follows. In Section 2, we provide a brief review of some basic concepts related to DT. Section 3 proposes the new learning algorithm in detail. The efficiency and efficacy of the new algorithm is verified through experiments with several public databases in Section 4. Section 5 is the conclusion.

\section{Review of Decision Trees}

\subsection{The Definition}

A decision tree (DT) is a directed graph with no cycles. There is one special node called root. We usually draw a DT with the root at the top (see Fig. 1). Each node (except the root) has exactly one node above it, which is called its parent. The nodes directly below a node are called its children. A node is called a terminal node if it dose not have any child. A node is non-terminal if it has at least one child. The node of a DT can be defined as a 5-tuple as follows:

$$
\text { node }=\{I, F, Y, N, L\}
$$

where $I$ is a unique number assigned to each node, $F$ is a test function that assigns a given input pattern to one of the children, $Y$ is a set of pointers to the children, $N=|Y|$ is the number of children or the size of $Y$, and $L$ is the class label of the node if it is a terminal node. For terminal nodes, $F$ is not defined and $Y$ is empty $(N=0)$. Usually, $L$ is not defined for non-terminal nodes. 
The process for recognizing an unknown pattern $x$ is as follows:

- Step 1: Set the root as the current node.

- Step 2: If the current node is a terminal node, assign $x$ with the class label of this node, and return; otherwise, find $i=F(x)$.

- Step 3: Set the $i$-th child as the current node, and return to Step 2.

\subsection{Induction of the DTs}

To induce a DT, it is assumed that a training set is available. Usually, the DT is induced by partitioning the training set recursively. This procedure involves three steps: 1) splitting nodes, 2) determining which nodes are terminal nodes, and 3) assigning class labels to terminal nodes.

To see a node is a terminal node or not, the simplest way is to check if all or most examples assigned to this node belong to the same class. If all or most examples are from the same class, the node is terminal, and its label is usually defined as the class label of the majority examples.

The purpose of splitting a node is to find a good test function $F$ for that node, so that the training examples assigned to this node can be partitioned into $N$ groups according to the test results. Here we need a measure to quantify the "goodness" of the test function. Many criteria have been proposed in the literature [2]-6]. For example, the criterion used in the well known induction algorithm $\mathrm{C} 4.5$ is the information gain ratio [6. It is known that the performance of a DT dose not appear to vary significantly over a wide range of criteria [2.

\subsection{Definition of the NNTrees}

An NNTree is a DT with each non-terminal node containing an ENN (see Fig. 1). To use an NNTree for learning with data streams, we define a node of an NNTree as follows:

$$
\text { node }=\{I, F, Y, N, L, W\}
$$

where $W$ is a sliding window to keep examples for retraining. The sliding window is nothing but a queue (or a first-in-first-out memory). The test function $F$ is now defined by an ENN. As the ENNs, we use three layer multilayer perceptrons (MLPs). There are $M$ inputs, $K$ hidden neurons and $N$ output neurons.

Using an NNTree, an example $x$ can be recognized as follows:

- Step 1: Set the root as the current node.

- Step 2: If the current node is a terminal node, assign $x$ with the class label of this node, and return; otherwise, find

$$
F(x)=i=\arg \max _{1 \leq k \leq N} o_{k}
$$

where $o_{k}$ is the $k$-th output of the ENN.

- Step 3: Set the $i$-th child as the current node, and return to Step 2. 


\section{Learning with Data Streams Using the NNTrees}

A direct method for learning with data streams is to fix the structure of the NNTree, and retrain the ENNs only. This method, however, cannot get good results because retraining the ENNs alone is not powerful enough to integrate new information effectively [10. To improve the learnability of an NNTree, we proposed a new algorithm in this paper. Fig. 2 shows the flow-chart of this algorithm. The learning process is described as follows:

- Step 0: Initialize the tree by putting the first training example into the sliding window $W$ of the root node, and define the class label of the root node $\mathrm{s}$ that of the first example. The tree now contains only one terminal node.

- Step 1: Set the current node as the root node and receive a training example $x$ with the class label label $(x)$ from the data stream.

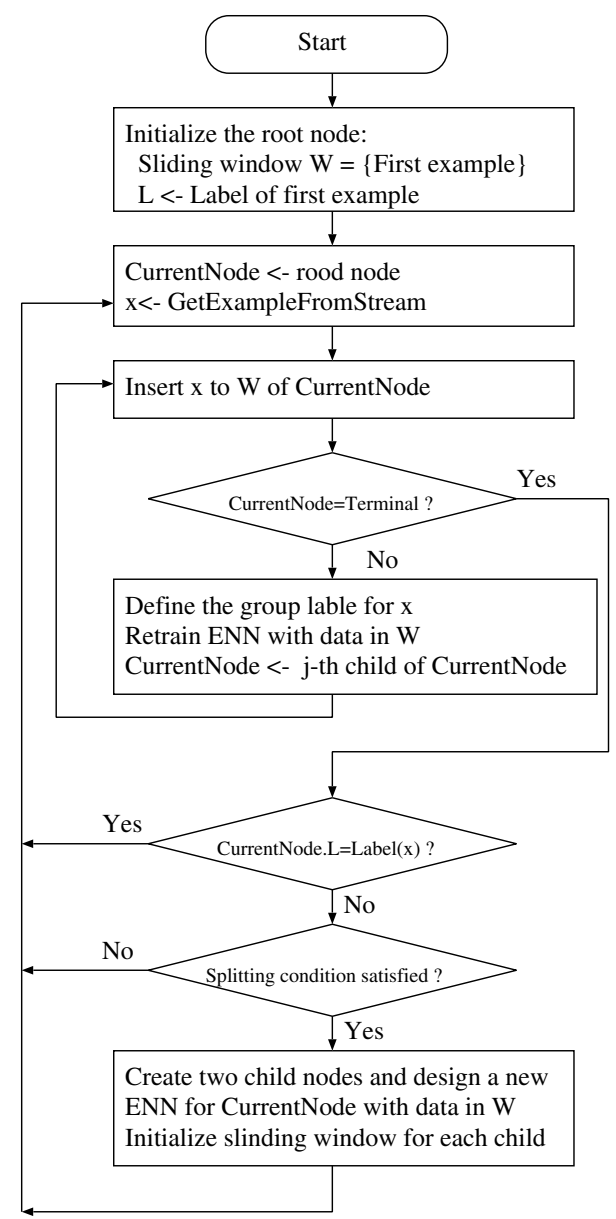

Fig. 2. Flow chart of the algorithm for learning with a data stream 
- Step 2: Insert $x$ into the sliding window $W$ of the current node.

- Step 3: If the current node is terminal, goto Step 5; otherwise, continue.

- Step 4: Define the group label of $x$ (see the definition given below), retrain the ENN using the back propagation (BP) algorithm for a limited number of epochs with data in $W$. After retraining, if the $j$-th output of the ENN is the maximum for $x$, set the current node as the $j$-th child of the current node, and return to Step 2.

- Step 5: If the $L$ value of the current node is $\operatorname{label}(x)$, return to Step 1; otherwise, continue (do nothing if $x$ can be recognized correctly).

- Step 6: If the splitting condition (see the definition given below) is not satisfied, return to Step 1; otherwise, continue.

- Step 7: Create $N$ child nodes and one ENN for the current node, and return to Step 1. The ENN is designed using BP algorithm with data in $W$. Based on the outputs of the ENN so designed, data in $W$ are split into $N$ parts. Data of the $i$-th part are put into the sliding window of the $i$-th child.

The group label of a example $x$, denoted by $g(x)$, is $i(i=1,2, \cdots, N)$ if this example is assigned to the $i$-th child node of the current node. Note that $g(x)$ is different from the class label of $x$. The class label label $(x)$ is given before learning (for supervised learning), while $g(x)$ must be determined during learning. In the proposed algorithm, the $g(x)$ is found as follows

1. Find $k=F(x)$, where $F(x)$ is the function realized by the ENN of the current node.

2. If $\exists y \in W_{k}$, where $W_{k}$ is the sliding window of the $k$-th child, such that label $(y)=\operatorname{lable}(x)$, then $g(x)=k$.

3. Else, if $\exists y \in W_{j}(j \neq k)$, such that label $(y)=\operatorname{lable}(x)$, then $g(x)=j$.

4. Else, $g(x)=k$.

Once the group label of $x$ is defined, we can retrain the ENN in the current node for a limited number of epochs. Note that the number of epochs is one when the ENN can assign $x$ to the correct child (in the sense defined by $g(x)$ ). Note also that if $x$ is a completely new example, i.e., we have not observed any example of the class label $(x)$ before, it will be assigned to the $k$-th child, where $k=F(x)$.

To prevent the tree from growing too fast, we use a splitting condition to determine when to split the node (in Step 6 of the learning algorithm). The condition used in our study is defined as follows:

$$
|S|>T \text { and } n_{\text {wrong }}>|S| \times s
$$

where $S$ is the set of examples assigned to the current node so far, $|S|$ is the size of $S, n_{\text {wrong }}$ is the number of mis-classified examples, $T$ is a threshold and $s$ is the splitting-rate. Generally speaking, $T$ and $s$ depend on the training set size and the number of classes. In this study, we just set $T$ to 30 and $s$ to 0.1 . With these values, the above condition can be read as "the current node will not be split if $|S|$ is less than 30, or if the mis-classified examples are less than $10 \%$ of $S$ ". Note that $S$ is not preserved during learning. Only $|S|$ is used. 
Table 1. Parameters of the Databases

\begin{tabular}{r|c|c|c}
\hline & Number of examples & Number of features & Number of classes \\
\hline cancer & 683 & 9 & 2 \\
\hline crx & 690 & 15 & 2 \\
\hline dermatology & 358 & 34 & 6 \\
\hline ionoshpere & 351 & 34 & 2 \\
\hline iris & 150 & 4 & 3 \\
\hline vehicle & 846 & 18 & 4 \\
\hline optdigits & 5620 & 64 & 10 \\
\hline pen-based & 10992 & 16 & 10 \\
\hline
\end{tabular}

When a terminal node is going to be split, we must design a new ENN for this node, and partition the data in the sliding window into $N$ groups based on this ENN. In this paper, the data are grouped as follows. Suppose that we want to partition the data in $W$ into $N$ sub-sets $S_{1}, S_{2}, \cdots, S_{N}$, which are initially empty sets. For any given example $x$,

1. If there is a $y \in S_{i}$, such that $\operatorname{label}(y)=\operatorname{label}(x)$, assign $x$ to $S_{i}$;

2. Else, if there is a $S_{i}$, such that $S_{i}=\Phi$, assign $x$ to $S_{i}$, where $\Phi$ is the empty set;

3. Else, find $y$, which is the nearest neighbor of $x$ in $\cup S_{i}$, and assign $x$ to the same sub-set as $y$, where $\cup$ represents the union of sets.

With the group labels so defined, we can design an ENN using the BP algorithm.

\section{Experimental Results}

To verify the efficiency of the proposed algorithm, we conducted several experiments using eight databases taken from the machine learning repository of the University of California at Irvine. The databases used include: cancer, crx, dermatology, ionosphere, iris, vehicle, optdigits and pen-based. These databases are actually real-life applications related to medical diagnosis, character recognition and so on. Parameters related to the databases are given in Table 1, 10-fold cross validation was used in the experiments. For learning with data streams, we select the training examples one-by-one without replacement, and provide it to the NNTree for retraining. The learning process will stop when all training examples are used.

The results of C4.5 [6] and ITI [1] are used for comparison. In the experiments, all parameters for $\mathrm{C} 4.5$ and ITI were default values. The main parameters related to the proposed method include: 1) the number of inputs $M$ for each ENN is the number of features; 2 ) the number of hidden neurons $K$ is 4 ; 3) the number of outputs $N$ is $2 / 4$ ) the maximum number of epochs for retraining the ENNs is $100 ; 5)$ the number of epochs for creating a new ENN is 1,000 ; and 6) the learning rate is 0.5 .

The computer used in the experiments is a Unix workstation with the following specifications: 1) the system model is Sun Java Workstation W1100z; 2) the 
main memory is $1 \mathrm{~GB} ; 3)$ the $\mathrm{CPU}$ is $1.8 \mathrm{GHz}$ AMD Opteron 144 (1MB cache); and 4) the OS is Solaris 9. All algorithms were run on the same computer.

Note that $\mathrm{C} 4.5$ is an off-line learning algorithm. It assumes that all data are available before learning, and thus the results should be better in terms of accuracy when compared with the results obtained by ITI or the proposed algorithm. However, if we consider the computational cost for learning with data streams, C4.5 will be the worst if the duration of the data stream is long. This is because a new tree must be induced for each new example. Compare ITI with the algorithm proposed here, ITI must preserve all information for restructuring the tree during learning. Thus, for long data streams, the computational cost of ITI will be higher than that of the proposed algorithm.

Tables [2]5] are the results obtained by C4.5, ITI and the proposed method with different sliding window sizes. In the tables, "Tree size" is the number of all nodes, "Error rate (\%)" is the rate for the test set, and "Computing time" is the time in seconds used for one run. For C4.5, only the results after pruning are provided here. For each result, there are two values. One is the average value over 10 runs, and another is the $95 \%$ confident interval.

\subsection{Discussion About the Accuracy}

As expected, the accuracy of the DTs (decision trees) induced by C4.5 are the best for 4 cases out of 8 . This is because that all data are available before learning. Although the authors of [1] claimed that ITI can obtain the same results as C4.5, the results given here do not support their claim. People may expect that NNTrees should outperform the DTs because they are more complex. This may be true for off-line learning. In this paper, however, we are talking about learning with data streams. That is, data are available only one by one. Results given here actually show that the NNTree based approach can resolve the plasticitystability dilemma to some extent because the NNTrees are comparable with the DTs induced by C4.5 or ITI. In 3 of the cases they are the best.

\subsection{Discussion About the Size}

In all cases, the number of nodes required by the NNTrees are smaller or much smaller than that of the DTs obtained by C4.5 or ITI. This is because an ENN is much more powerful than an axis-parallel hyperplane. Usually, one ENN can replace many hyperplanes for making the same decision. However, the tree size alone cannot be used to measure the complexity of the system. If we count the number of free parameters, the NNTrees are actually more complex. This increase in complexity is the main reason why NNTrees are able to learning with data streams.

\subsection{Discussion About the Computational Cost}

The main computational costs of the proposed algorithm include: 1) cost for computing the output of the network for each example, 2) cost for updating the 
Table 2. Results of C4.5

\begin{tabular}{r|r|r|r}
\hline Database & Tree size & Error rate (\%) & Computing Time \\
\hline cancer & $23.2 \pm 10.8$ & $4.8 \pm 4.3$ & $0.021 \pm 0.002$ \\
\hline crx & $30.3 \pm 24.3$ & $14.6 \pm 7.2$ & $0.035 \pm 0.003$ \\
\hline dermatology & $32.6 \pm 6.9$ & $6.4 \pm 6.8$ & $0.020 \pm 0.003$ \\
\hline ionoshpere & $27.2 \pm 6.5$ & $\mathbf{1 0 . 8} \pm \mathbf{8 . 7}$ & $0.090 \pm 0.011$ \\
\hline iris & $8.2 \pm 2.0$ & $\mathbf{4 . 0} \pm \mathbf{6 . 8}$ & $0.016 \pm 0.002$ \\
\hline vehicle & $136.0 \pm 45.5$ & $\mathbf{2 8 . 5} \pm \mathbf{8 . 5}$ & $0.078 \pm 0.010$ \\
\hline optdigits & $410.0 \pm 29.4$ & $9.4 \pm 2.1$ & $0.966 \pm 0.061$ \\
\hline pen-based & $380.4 \pm 23.8$ & $\mathbf{3 . 5} \pm \mathbf{1 . 0}$ & $0.687 \pm 0.011$ \\
\hline
\end{tabular}

Table 3. Results of ITI

\begin{tabular}{r|r|r|r}
\hline Database & Tree size & Error rate (\%) & Computing Time \\
\hline cancer & $18.4 \pm 2.2$ & $4.6 \pm 2.5$ & $0.115 \pm 0.017$ \\
\hline crx & $28.6 \pm 4.4$ & $\mathbf{1 3 . 5} \pm \mathbf{3 . 4 6}$ & $0.618 \pm 0.039$ \\
\hline dermatology & $37.0 \pm 3.0$ & $6.7 \pm 3.75$ & $0.582 \pm 0.092$ \\
\hline ionoshpere & $19.2 \pm 2.6$ & $12.8 \pm 6.17$ & $1.194 \pm 0.096$ \\
\hline iris & $5.8 \pm 0.6$ & $3.8 \pm 4.4$ & $0.012 \pm 0.004$ \\
\hline vehicle & $118.8 \pm 12.2$ & $28.59 \pm 5.14$ & $4.933 \pm 0.445$ \\
\hline optdigits & $919.2 \pm 53.8$ & $22.3 \pm 1.6$ & $510.639 \pm 65.885$ \\
\hline pen-based & $648.2 \pm 28.6$ & $5.8 \pm 0.9$ & $480.042 \pm 11.180$ \\
\hline
\end{tabular}

Table 4. Results of proposed method, window size $=50$

\begin{tabular}{r|r|r|r}
\hline Database & Tree size & Error rate (\%) & Computing Time \\
\hline cancer & $3.20 \pm 0.63$ & $\mathbf{4 . 1} \pm \mathbf{1 . 8}$ & $0.383 \pm 0.273$ \\
\hline crx & $17.40 \pm 3.86$ & $17.4 \pm 4.1$ & $5.604 \pm 2.190$ \\
\hline dermatology & $11.80 \pm 1.03$ & $3.6 \pm 3.2$ & $0.321 \pm 0.108$ \\
\hline ionoshpere & $9.00 \pm 2.31$ & $13.6 \pm 4.9$ & $1.789 \pm 1.040$ \\
\hline iris & $6.00 \pm 1.05$ & $5.0 \pm 5.7$ & $0.352 \pm 0.237$ \\
\hline vehicle & $59.40 \pm 9.18$ & $31.3 \pm 4.6$ & $16.699 \pm 3.113$ \\
\hline optdigits & $41.00 \pm 5.25$ & $7.8 \pm 1.9$ & $24.052 \pm 5.316$ \\
\hline pen-based & $68.00 \pm 11.78$ & $7.1 \pm 1.9$ & $29.361 \pm 3.532$ \\
\hline
\end{tabular}

Table 5. Results of proposed method, window size $=100$

\begin{tabular}{r|r|r|r}
\hline Database & Tree size & Error rate (\%) & Computing Time \\
\hline cancer & $3.20 \pm 0.63$ & $4.5 \pm 3.1$ & $2.385 \pm 1.485$ \\
\hline crx & $14.20 \pm 2.53$ & $16.7 \pm 3.6$ & $14.614 \pm 2.474$ \\
\hline dermatology & $11.60 \pm 0.97$ & $\mathbf{2 . 2} \pm \mathbf{2 . 9}$ & $0.564 \pm 0.257$ \\
\hline ionoshpere & $8.40 \pm 2.32$ & $11.9 \pm 6.7$ & $6.415 \pm 2.763$ \\
\hline iris & $5.60 \pm 0.97$ & $4.4 \pm 5.2$ & $0.803 \pm 0.477$ \\
\hline vehicle & $59.20 \pm 9.11$ & $30.0 \pm 3.5$ & $41.468 \pm 8.224$ \\
\hline optdigits & $39.80 \pm 7.07$ & $\mathbf{6 . 9} \pm \mathbf{1 . 6}$ & $82.543 \pm 22.161$ \\
\hline pen-based & $54.00 \pm 9.30$ & $5.9 \pm 1.3$ & $88.233 \pm 15.901$ \\
\hline
\end{tabular}


weights, 3) cost for all epochs, 4) cost for all nodes on the search path, and 5) cost for all training examples. Put all these costs together, we have the total cost:

$$
\operatorname{Cost}(\text { Proposed })=O\left(M \times K \times N_{w} \times N_{e} \times \log T \times N_{t}\right)
$$

where $M$ is the dimensionality of the feature space, $K$ is the number of hidden neurons, $N_{w}$ is the number of examples in the sliding window, $T$ is the size of the current tree, and $N_{t}$ is the length of the data stream.

On the other hand, the computational cost of $\mathrm{C} 4.5$ is

$$
\begin{aligned}
\operatorname{Cost}(C 4.5) & =O\left[M \times m \times \log T \times\left(1+2+\ldots+N_{t}\right)\right] \\
& =O\left(M \times m \times \log T \times N_{t}^{2}\right)
\end{aligned}
$$

where $m$ is the possible number of values of a feature and $T$ is the size of the current tree. Note that the computational costs in all levels (not node) should be almost the same. Therefore, instead of using $(T-1) / 2$, which is the number of non-terminal nodes, we use $\log T$ here. Note also that in the worst case, $m=N_{t}$, and thus

$$
\operatorname{Cost}(C 4.5)=O\left(M \times \log T \times N_{t}^{3}\right)
$$

Based on the above considerations, we can get the ratio between the cost of the proposed method and that of $\mathrm{C} 4.5$ :

$$
\begin{aligned}
R & =\frac{O\left(M \times K \times N_{w} \times N_{e} \times \log T \times N_{t}\right)}{O\left(M \times \log T \times N_{t}^{3}\right)} \\
& =\frac{O\left(K \times N_{w} \times N_{e}\right)}{O\left(N_{t}^{2}\right)}
\end{aligned}
$$

In the experiments, $N_{e}=100, K=4$, and $N_{w}=50$, or 100 . Thus, for long data streams, C4.5 will be much more time-consuming than the proposed method. Note that in Table 2, the computing time is only for the case $n_{t}=N_{t}$. If the data are provided one by one, the computing time should times the factor $N_{t}\left(N_{t}+1\right) / 2$.

As for the algorithm ITI, we do not know exactly the computational cost. In fact, even the authors of [1] did not provide an analysis of the computational cost. However, from the results given in Tables 3.5] we can see that for long data streams, ITI is more time consuming than the proposed method. This is also what we have expected.

\section{Conclusion}

In this paper, we have studied learning with data streams using the NNTrees, and proposed a new algorithm. The efficiency and efficacy of the algorithm have been verified through experiments with eight public databases. Note that all databases used in the experiments are not "endless data streams". We will do 
more experiments in the future using longer data streams to verify the usefulness of the proposed algorithm. In fact, we are trying to apply the proposed algorithm to stock prediction. More results will be reported later.

\section{Acknowledgments}

This research is supported in part by the Grants-in-Aid for Scientific Research of Japan Society for the Promotion of Science (JSPS), No. 17500148.

\section{References}

1. P. E. Utgoff, N. C. Berkman and J. A. Clouse, "Decision tree induction based on efficient tree restructuring," Machine Learning, vol. 29, pp. 5-44, 1997.

2. L. Brieman, J. H. Friedman, R. A. Olshen and C. J. Stong, Classification and regression trees, Belmont, CA: Wadsworth, 1984.

3. S. B. Gelfand, C. S. Ravishankar, and E. J. Delp, "An iterative growing and Pruning algorithm for classification tree design," IEEE Trans. on Pattern Analysis and Machine Intelligence, 13-2 (1991) 163-174.

4. E. G. Henrichon, JR. and K. S. Fu, "A non-parametric partitioning procedure for pattern classification," IEEE Trans. on Computers, 18-7 (1969) 614-624.

5. L. Hyafil, "Construction optimal binary decision trees is NP-complete," Information Processing Letters, 5-1 (1976) 15-17.

6. J. R. Quinlan, C4.5: Programs for Machine Learning, Morgan Kaufmann Publishers, 1993.

7. Q. F. Zhao, "Evolutionary design of neural network tree - integration of decision tree, neural network and GA," Proc. IEEE Congress on Evolutionary Computation, (Korea, Seoul, 2001). 240-244.

8. Q. F. Zhao, "Training and retraining of neural network trees," Proc. INNS-IEEE International Joint Conference on Neural Networks, (Washington DC, 2001) 726731.

9. Q. F. Zhao, "Modeling and evolutionary learning of modular neural networks," Proc. The 6-th International Symposium on Artificial Life and Robotics, (2001) 508-511.

10. T. Takeda and Q. F. Zhao, "A two step algorithm for designing small neural network trees," Proc. IEEE International Conference on Neural Networks and Signal Processing (ICNNSP03), (China, Nanjing, 2003) 513-517.

11. Q. F. Zhao, "Design Smart NNTrees Based on the $R^{4}$-rule," Proc. of the IEEE 19th International Conference on Advanced Information Networking and Applications (AINA2005), Vol. 2, pp. 547-551, 2005. 